\title{
Detection of nivalenol and deoxynivalenol chemotypes produced by Fusarium graminearum species complex isolated from barley in Iran using specific PCR assays
}

\author{
Khosrow Chehri*, Rasoul Godini \\ Department of Biology, Faculty of Science, Razi University, Kermanshah, Iran
}

Vol. 57, No. 3: 237-242, 2017

DOI: 10.1515/jppr-2017-0033

Received: September 12, 2017

Accepted: August 17, 2017

*Corresponding address:

khchehri@gmail.com

\begin{abstract}
In order to identify trichothecenes chemotypes produced by Fusarium graminearum species complex (FGSC) isolated from barley, 68 barley samples were collected from markets in Kermanshah and Hamedan provinces, Iran. Thirty-one Fusarium isolates were obtained from grains and morphologically classified into three species FGSC (14), F. equiseti (9), and F. proliferatum (8). The identification of the members of FGSC was confirmed molecularly using Fg16F/Fg16R primers. Fusarium asiaticum isolates (4) were distinguished from other FGSC using Fg6CTPSf177/Fg16R primers. Polymerase chain reaction-based (PCRbased) detection of mycotoxin-synthesis-pathway gene was also used to determine the potential of the analysed strains to produce deoxynivalenol (DON), 15-acetyldeoxynivalenol (15-AcDON), 3-acetyldeoxynivalenol (3-AcDON), and nivalenol (NIV). Of 14 tested isolates, 10 and 4 isolates belonged to DON and NIV chemotype, respectively. Also, the results of DON chemotype survey using specific primers MinusTri7F/R and Tri315F/R showed 1 and 9 isolates produced 3-AcDON and 15-AcDON, respectively. These results show that DON was the most common chemotype in western Iran. To our knowledge, this is the first report on 15-AcDON, 3-AcDON, and NIV isolated from barley in Iran.
\end{abstract}

Key words: barley, deoxynivalenol, FGSC, Fusarium graminearum, PCR, trichothecenes

\section{Introduction}

Many strategic agricultural crops such as members of the Poaceae family are subject to pathogenic fungi, particularly species from the Fuarium genus (Waalwijk et al. 2003). One well-known disease in Poaceae species such as barley, rye and wheat is Fusarium head blight (FHB). FHB outbreaks are common on many continents including North and South America, Europe, Asia and Australia and impose extremely high economic losses (McMullen et al. 1997; Obanor et al. 2013). Fusarium head blight agents invade heads and kernels of small cereal plants and contaminate them with secretion of mycotoxins (Boutigny et al. 2011; Postic et al. 2012). In many part of the world, contamination of crops with mycotoxins is a major problem in the agricultural industry. Mycotoxins are secondary metabolites produced by some species of fungi, such as Fusarium graminearum species complex (FGSC) members, which are responsible for hazardous effects on animals as well as humans. Mycotoxins can enter the food-chain through direct consummation of infected food either by humans or livestock (Postic et al. 2012).

Studies show that outbreaks of FHB have been increasing throughout the world over the past decades (Obanor et al. 2013). FHB has been identified worldwide and is caused by multiple agents from Fusarium spp. which can be very diverse in different countries. Fusarium culmorum, F. poae, and F. avenaceum are observed in many European countries while F. asiaticum is observed in Asia (Waalwijk et al. 2003). Fusarium graminearum and $F$. asiaticum, members of the FGSC, are the most important agents of FHB. Fusarium graminearum are predominant in Asia, Australia, Europe, and America (Akinsanmi et al. 2004; Somma 
et al. 2014), whereas F. asiaticum is the major agent of FHB in many Asian countries such as China, Korea, Japan and Nepal (Zhang et al. 2007; Karugia et al. 2009; Lee et al. 2009; Sarver et al. 2011).

FGSC possesses extremely important species which are very widespread. At least 15 distinct lineages which are morphologically indistinguishable have been identified by molecular studies (Sarver et al. 2011). Some members of FGSC produce mycotoxins type $B$ trichothecenes that can be classified into three strain-specific chemotypes, including the nivalenol (NIV), 3-acetyldeoxynivalenol (3-AcDON) and 15-acetyldeoxynivalenol (15-AcDON) chemotypes. The NIV chemotype produces nivalenol, and 3-AcDON and 15-AcDON are acetylated forms of deoxynivalenol (DON) (Miller et al. 1991; Boutigny et al. 2011). For chemotype identification, there is a rapid procedure based on the amplification of specific genes encoding for proteins involved in mycotoxin biosynthesis. Genes such as: TRI2, TRI3, TRI5, TRI6, TRI7, TRI13 and TRI101 are encoded for biosynthesis of trichothecene and can be assayed by PCR to identify the ability of trichothecene biosynthesis and also chemotypes of the fungi (Chandler et al. 2003; Haratian et al. 2008; Sampietro et al. 2010; Yoruk et al. 2016).

Barley is a strategic plant cultivated on more than 190,000 and 120,000 ha in just Kermanshah and Hamedan provinces, respectively. However, very few studies about FGSC invading barley and their chemotypes have been done. The aims of this study were firstly, the identification of the Fusarium spp. isolated from barley in Iran in 2012, 2013, and 2014 using morphological and molecular methods by speciesspecific PCR and secondly, determination of NIV and 3-AcDON and 15-AcDON chemotypes of the isolates.

\section{Materials and Methods}

Barley grains were purchased from agricultural markets in Kermanshah and Hamedan provinces. To isolate fusaria, barley grains were randomly hand-selected and placed on water agar amended with peptone-pentachloronitro benzene plates (Nash and Snyder 1962) and incubated at $25^{\circ} \mathrm{C}$ for 4 days. The single spores of Fusarium grown on water agar medium were transferred onto potato dextrose agar and carnation leaf agar media to prepare pure colonies. In order to identify species, macroscopic and microscopic characteristics were studied and the results were compared to the species descriptions of Leslie and Summerell (2006).

DNA was extracted from all FGSC isolates and analysed. Briefly, Fusarium isolates were cultured in potato dextrose broth (Sigma) medium, shaking at $150 \mathrm{rpm}$ at $25 \pm 2^{\circ} \mathrm{C}$ for 5 days. Mycelia were harvested by filtration through Whatman paper 1 and freezedried for $20 \mathrm{~h}$. DNA was extracted using a DNeasy Plant Mini Kit (Qiagen) according to the manufacturer's protocol.

All isolates were investigated by species-specific PCR assay to identify F. asiatecum from other members of FGSC. Previously published primer pairs were used for this step (Table 1). Amplification reactions were performed in a total volume of $25 \mu \mathrm{l}$, by mixing

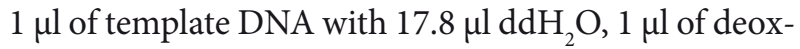
ynucleotide triphosphate (dNTP) (Promega) - $1 \mu \mathrm{l}$ of each primer, $0.2 \mu \mathrm{l}$ of Taq DNA polymerase (Promega), $0.5 \mu \mathrm{l}$ of $\mathrm{MgCl}_{2}$ (Promega) and $2.5 \mu \mathrm{l}$ of PCR 5X reaction buffer (Promega, Madison, Wl, USA). PCR amplification was done in the Peltier Thermal Cycler, PTC- $100^{\circ}$ (MJ Research, Inc. USA) with the following programs: an initial denaturation step at $94^{\circ} \mathrm{C}$ for $5 \mathrm{~min}, 35$ cycles at $94^{\circ} \mathrm{C}(1 \mathrm{~min}) / 56^{\circ} \mathrm{C}(1 \mathrm{~min}) / 72^{\circ} \mathrm{C}$

Table 1. Species-specific primers sequences and size of PCR products

\begin{tabular}{llcc}
\hline \multicolumn{1}{c}{ Primer name } & \multicolumn{1}{c}{ Sequence 5'-3' } & Product size [bp] & Source \\
\hline Fg16F & CTCCGGATATGTTGCGTCAA & $400-500$ & Nicholson et al. (1998) \\
Fg16R & GGTAGGTATCCGACATGGCAA & & \\
Fg6CTPSf177 & GTCTCACTTCAAGCCA & 162 & Yang et al. (2008) \\
FgCTPSrR306 & CCTTGGTCATCCATAGAG & & \\
Tri315F & CTCGCTGAAGTTGGACGTAA & 864 & Jennings et al. (2004) \\
Tri315R & GTCTATGCTCTCAACGGACAAC & & \\
MinusTri7F & TGGATGAATGACTTGAGTTGACA & 483 & Ward et al. (2002) \\
MinusTri7R & AAAGCCTTCATTCACAGCC & & \\
ToxP1 & GCCGTGGGGATAAAAGTCAAA & $300-360$ & Li et al. (2005) \\
ToxP2 & TGACAAGTCCGGTCGCACTAGCA & & Nicholson et al. (1998) \\
\hline
\end{tabular}


(3 $\mathrm{min})$, and a final extension step at $72^{\circ} \mathrm{C}$ for $10 \mathrm{~min}$. The PCR products were visualized by $1 \mathrm{X}$ Tris/Borate/ EDTA buffer (TBE) electrophoresis in ethidium-bromide-stained, $1 \%$ agarose gel.

Trichothecenes chemotypes of Fusarium spp. isolates were determined by PCR-based molecular analyses of the mycotoxin-synthesis pathway genes of NIV, DON, 15-AcDON, and 3-AcDON. ToxP1/P2 primers were designed for Tri5 and Tri6 gene sequences and used to identify the isolates able to produce NIV and DON. Tri315F/R and MinusTri7F/R were used to make 864 bp specific fragments for $15-\mathrm{AcDON}$ and $483 \mathrm{bp}$ specific fragments for 3-AcDON, respectively (Table 1).

Amplification reactions were made in a total volume of $25 \mu \mathrm{l}$ for each of the isolates which contained $4 \mu \mathrm{l}$ 10X buffer (Promega, Madison, WI, USA), $2 \mathrm{mM} \mathrm{MgCl}_{2}, 0.2 \mathrm{mM}$ deoxynucleotide triphosphate (dNTP) (Promega), $0.4 \mu \mathrm{M}$ of each primer, 0.75 units of Taq DNA polymerase (Promega), and 50 ng of template DNA. PCR amplification was carried out in the Peltier Thermal Cycler, PTC-100 (MJ Research, Inc. USA) according to temperature profiles described by Lenc et al. (2008) and Lenart et al. (2013). To visualize the PCR products, $1 \mathrm{X}$ TBE electrophoresis in ethidium-bromide-stained and 1\% agarose gel were used.

\section{Results}

Sixty-eight barley samples purchased from markets in Kermanshah and Hamedan provinces were analyzed for the occurrence of FGSC and trichothecenes chemotypes. Twenty-one samples were found positive for the Fusarium species. A total of 31 isolates were obtained. The isolates were classified into three species FGSC (14), F. equiseti (9), and F. proliferatum (8) based on morphological characters (Table 2).

Members of FGSC were distinguished molecularly using Fg16F/Fg16R primers. The primers Fg16F/Fg16R produced fragments of $450 \mathrm{bp}$ in 14 isolates. Based on morphological features they were identified as FGSC. Therefore, our morphological studies were confirmed by molecular outputs. Fg6CTPSf177/FgCTPSrR306 primers produced fragments of 162 bp only in 4 isolates which belonged to F. asiaticum (Fig. 1).

Four PCR assays (DON, 15-AcDON, 3-AcDON, and NIV) were used for identification of chemotypes of the FGSC isolates. ToxP1/P2 primers that were designed based on Tri5 and Tri6 gene sequences were used to identify DON and NIV producer isolates. ToxP1/P2 primers produced fragments of 300 and $360 \mathrm{bp}$ in 10 and 4 isolates, respectively (Fig. 2). Specific primers Tri315F/R and MinusTri7F/R which are designed based on Tri3 and Tri7 gene sequences, respectively, were used to identify 15-AcDON and 3-AcDON chemotypes. Tri315F/R and MinusTri7F/R primers produced fragments of $864 \mathrm{bp}$ and 483 bp in 9 and 1 isolates, respectively (Fig. 3).

PCR results showed that $15-A c D O N$-producing strains existed in all cities in western Iran, except Bisotun, and only one 3-AcDON-producing strain was observed in Kamyaran. NIV-producing strains were observed in the cities of Sarpole-Zahab, Kamyaran, Asad Abad, and Bisotun (Table 2).

Table 2. Place of sample collection, Fusarium spp., frequencies of potentially toxigenic (deoxynivalenol - DON and nivalenol - NIV) strains isolated from barley in Iran

\begin{tabular}{|c|c|c|c|c|c|}
\hline $\begin{array}{l}\text { Place of sample } \\
\text { collection }\end{array}$ & $\begin{array}{c}\text { No. of barley } \\
\text { samples } \\
\text { (No. of infected } \\
\text { samples } \\
\text { with Fusarium spp.) }\end{array}$ & $\begin{array}{l}\text { Fusarium spp. } \\
\text { identified }\end{array}$ & $\begin{array}{l}\text { 3-AcDON- } \\
\text {-producing strain }\end{array}$ & $\begin{array}{l}15-A c D O N- \\
\text {-producing strains }\end{array}$ & $\begin{array}{l}\text { NIV-producing } \\
\text { strains }\end{array}$ \\
\hline Sarpol-e Zohab & $7(2)$ & F. eq, F. gr, F. as, F. pr & - & F. $g r(1)$ & F. as (1) \\
\hline Kamiaran & $7(3)$ & F. eq, F. gr, F. pr & F. $g r(1)$ & F. $g r(1)$ & F. $g r(1)$ \\
\hline Ravansar & $7(3)$ & F. eq, F. gr, F.pr & - & F.gr(1) & - \\
\hline Sahneh & $7(2)$ & F. gr, F.pr & - & F. $g r(1)$ & - \\
\hline Gilan-e Gharb & $7(1)$ & F. eq, F. gr, F. as & - & F. as (1) & - \\
\hline Kermanshah & $7(2)$ & F.gr, F.eq & - & F. $g r(1)$ & - \\
\hline Bisotun & $7(2)$ & F. eq, F.gr, F.pr & - & - & F. $g r(1)$ \\
\hline Hamedan & $7(2)$ & F. eq, F. as, F. pr & - & F. as (1) & - \\
\hline Ghorveh & $6(2)$ & F. eq, F. gr, F. as, F. pr & - & F. as (1) & - \\
\hline Asad Abad & $6(2)$ & F. eq, F. gr, F.pr & - & F. $g r(1)$ & F. $g r(1)$ \\
\hline
\end{tabular}

F. eq $=$ F. equiseti, F. gr $=$ F. graminearum, F. as $=F$. asiaticum, F. pr $=$ F. proliferatum 


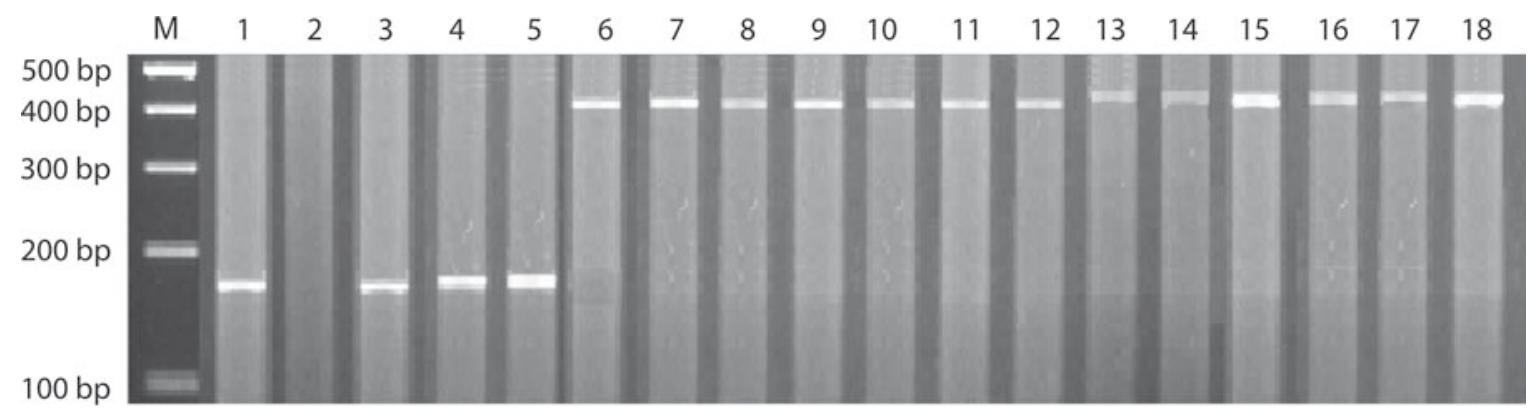

Fig. 1. PCR products obtained with specific primer pairs Fg16F/Fg16R (band, $450 \mathrm{bp}$ ) and Fg6CTPSf177/FgCTPSrR306 (band, 162 bp) from 14 isolates of FGSC. Lane M: GeneRuler DNA Ladder Mix, 100-10,000 bp Ladder. (1 = FGSCB1, 2 = Fusarium proliferatum, 3 = FGSCB2, 4 = FGSCB3, 5 = FGSCB4) amplified with primers Fg6CTPSf177 and FgCTPSrR306. (6 = FGSCB1, 7 = FGSCB2, 8 = FGSCB3, 9 = FGSCB4, $10=$ FGSCB5, $11=$ FGSCB6, $12=$ FGSCB7, $13=$ FGSCB8, $14=$ FGSCB9, $15=$ FGSCB10, $16=$ FGSCB11, $17=$ FGSCB12, $18=$ FGSCB13) amplified with primers pairs Fg16F/Fg16R

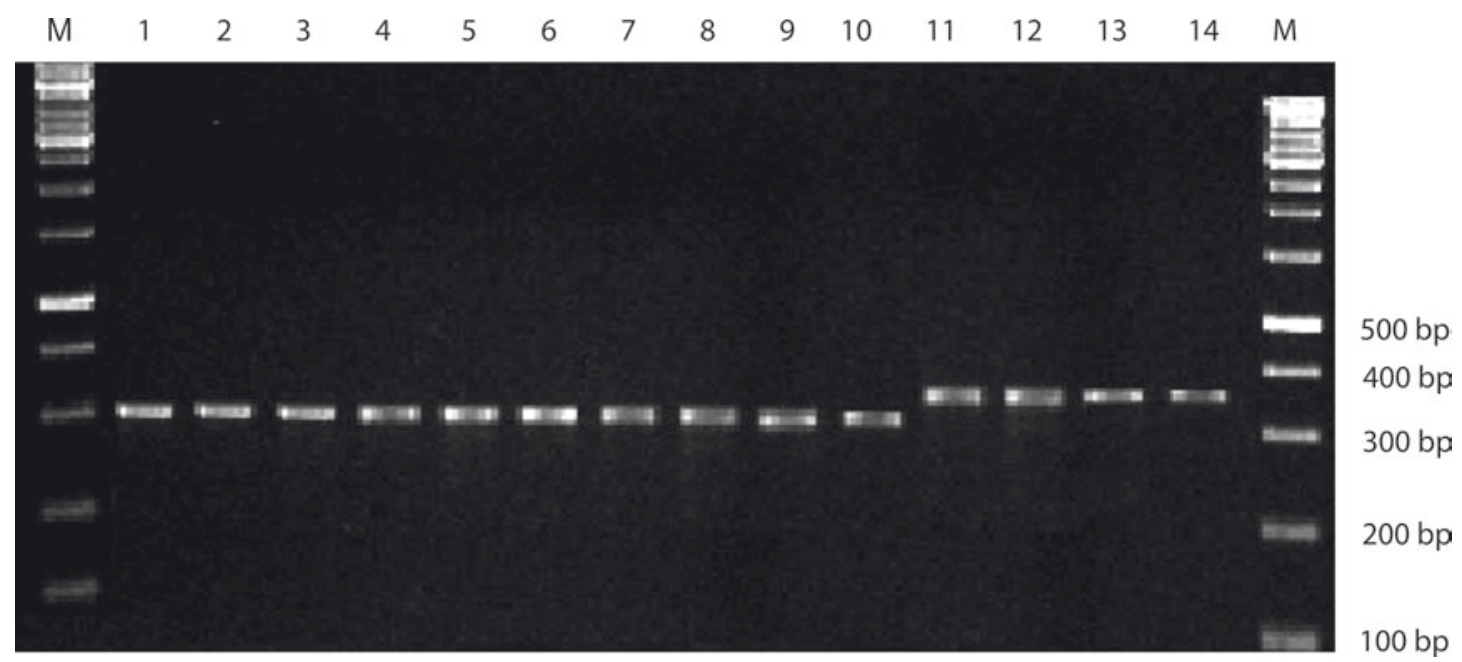

Fig. 2. PCR detection of deoxynivalenol (DON) (band, $300 \mathrm{bp}$ ) and nivalenol (NIV) (band, $360 \mathrm{bp}$ ) obtained with specific primer pairs of ToxP1/P2. Lane M: GeneRuler DNA Ladder Mix, 100-10,000 bp Ladder. Lanes $1=$ FGSCB2, $2=$ FGSCB3, $3=$ FGSCB4, 4 = FGSCB51, $5=$ FGSCB6, 6 = FGSCB8, 7 = FGSCB9, $8=$ FGSCB10, $9=$ FGSCB11, and $10=$ FGSCB12 were positively identified as DON genotypes, and lanes $11=$ FGSCB1, $12=$ FGSCB7, $13=$ FGSCB13, and $14=$ FGSC14 were positively identified as NIV genotypes

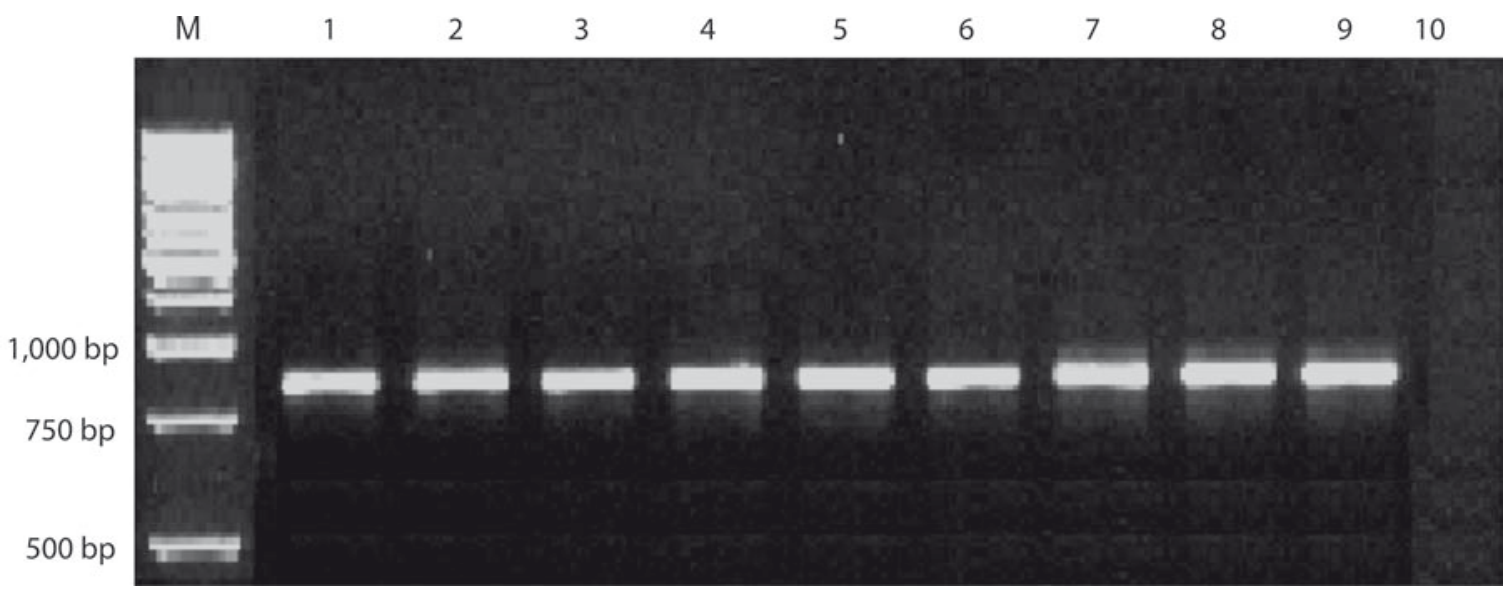

Fig. 3. $P C R$ detection of $15-A c D O N$ (band, 864 bp) obtained with specific primer pairs of Tri315F/R. Lane M: GeneRuler $1 \mathrm{~kb}$ DNA Ladder. Lanes 1 = FGSCB2, 2 = FGSCB3, 3 = FGSCB4, 4 = FGSCB51, 5 = FGSCB6, 6 = FGSCB8, 7 = FGSCB9, 8 = FGSCB10, and $9=\mathrm{FGSCB} 11$ were positively identified as $15-\mathrm{ACDON}$ genotypes 


\section{Discussion}

Barley is classified as a strategic crop which is cultivated in most parts of the world and is a typical host for FHB (Yang et al. 2008). Previous studies have shown that species causing barley FHB can vary in different part of the world (Kim et al. 1993; Goswami and Kistler 2004). However, there have been only limited attempts to classify fungal isolates associated with barley in Iran. Our morphological studies identified 31 isolates in the barley samples from western Iran which belong to three species including FGSC, F. equiseti, and F. proliferatum. FGSC has at least 15 morphologically unidentifiable species, including F. graminearum sensu strico and F. asiaticum which are the casual agents of FHB global epidemics (Sarver et al. 2011). Both species of $F$. graminearum and $F$. asiaticum have been reported from wheat in northern and northwestern areas of Iran (Davari et al. 2012). In the present study, for the first time, both of these species were isolated from barley cultivated in Iran and identified through molecular analyses based species-specific PCR. We molecularly detected that $F$. asiaticum constitute about $30 \%$ (4 out of 14 isolates) of the isolates belonging to FGSC. Previous studies showed that $F$ asiaticum was commonly isolated in warmer areas (above $22^{\circ} \mathrm{C}$ ) and the majority of $F$ graminearum sensu strico was obtained from cooler areas $\left(22^{\circ} \mathrm{C}\right.$ or lower) (Qu et al. 2008; Backhouse 2014). In our study, F. graminearum sensu strico was obtained from almost all regions in western Iran and F. asiaticum was mainly isolated from the warmer regions of the same area.

FGSC members produce thrichothecene mycotoxins which can be determined using PCR based analysis. Previous studies identified chemotypes of FGSC members by considering genes related to biosynthesis of the compounds (Jennings et al. 2004; Quarta et al. 2006; Sampietro et al. 2012; Zhang et al. 2012). There are few reports based on geographical distribution of FGSC chemotypes and their host ranges in different regions of Iran. Haratian et al. (2008) examined NIV and DON chemotypes of isolates collected from different fields in Mazandaran province, in northern Iran. In the present study, we used specific primers to identify the NIV and DON trichothecenes chemotypes among the molecularly confirmed FGSC isolates. Our results revealed that there were more DON chemotypes than NIV chemotypes. Moreover, investigation of the acetylated form of DON showed that 9 and 1 isolates were classified as 15AcDON and 3-AcDON chemotypes, respectively. To our knowledge, these results are presented for the first time for barley cultivated in Iran.

In conclusion, our findings showed that speciesspecific primers can be used for rapid detection of NIV and DON trichothecenes chemotypes in infected tissues and 15-AcDON was the most abundant chemotype in western Iran.

\section{Acknowledgements}

Khosrow Chehri acknowledges the Razi University, Kermanshah, Iran for providing necessary facilities to carry out this research.

\section{References}

Akinsanmi O.A., Mitter V., Simpfendorfer S., Backhouse D., Chakraborty S. 2004. Identity and pathogenicity of Fusarium spp. isolated from wheat fields in Queensland and northern New South Wales. Australian Journal of Agricultural Research 55 (1): 97-107. DOI: https://doi.org/10.1071/ $\operatorname{ar} 03090$

Backhouse D. 2014. Global distribution of Fusarium graminearum, F. asiaticum and F. boothii from wheat in relation to climate. European Journal of Plant Pathology 139 (1): 161-173. DOI: https://doi.org/10.1007/s10658-013-0374-5

Boutigny A.L., Ward T.J., Van Coller G.J., Flett B., Lamprecht S.C., O’Donnell K., Viljoen A. 2011. Analysis of the Fusarium graminearum species complex from wheat, barley and maize in South Africa provides evidence of species-specific differences in host preference. Fungal Genetics and Biology 48 (9): 914-920.

Chandler E.A., Duncan R.S., Thomsett M.A., Nicholson P. 2003. Development of PCR assays to tri7 and tri13 and characterisation of chemotypes of Fusarium graminearum, Fusarium culmorum and Fusarium cerealis. Physiological and Molecular Plant Pathology 62 (6): 355-367. DOI: https://doi. org/10.1016/s0885-5765(03)00092-4

Davari M., van Diepeningen A.D., Babai-Ahari A., Arzanlou M., Najafzadeh M.J., van der Lee T.A., de Hoog G.S. 2012. Rapid identification of Fusarium graminearum species complex using Rolling Circle Amplification (RCA). Journal of Microbiological Methods 89 (1): 63-70. DOI: https://doi. org/10.1016/j.mimet.2012.01.017

Goswami R.S., Kistler H.C. 2004. Heading for disaster: Fusarium graminearum on cereal crops. Molecular Plant Pathology 5 (6): 515-525. DOI: https://doi.org/10.1111/j.13643703.2004.00252.x

Haratian M., Sharifnabi B., Alizadeh A., Safaie N. 2008. PCR analysis of the Tri13 gene to determine the genetic potential of Fusarium graminearum isolates from Iran to produce nivalenol and deoxynivalenol. Mycopathologia 166 (2): 109-116. DOI: https://doi.org/10.1007/s11046-008-9127-y

Jennings P., Coates M.E., Turner J.A., Chandler E.A., Nicholson P. 2004. Determination of deoxynivalenol and nivalenol chemotypes of Fusarium culmorum isolates from England and Wales by PCR assay. Plant Pathology 53 (2): 182-190. DOI: https://doi.org/10.1111/j.0032-0862.2004.00985.x

Karugia G.W., Suga H., Gale L.R., Nakajima T., Tomimura K., Hyakumachi M. 2009. Population structure of the Fusarium graminearum species complex from a single Japanese wheat field sampled in two consecutive years. Plant Disease 93 (2): 170-174. DOI: https://doi.org/10.1094/pdis-93-2-0170

Kim J-C., Kang H-J., Lee D.H., Lee Y.W., Yoshizawa T. 1993. Natural occurrence of Fusarium mycotoxins (trichothecenes and zearalenone) in barley and maize in Korea. Applied and Environmental Microbiology 59 (11): 3798-3802.

Lee J., Chang I.Y., Kim H., Yun S.H., Leslie J.F., Lee Y.W. 2009. Genetic diversity and fitness of Fusarium graminearum populations from rice in Korea. Applied and Environmen- 
tal Microbiology 75 (10): 3289-3295. DOI: https://doi. org/10.1128/aem.02287-08

Lenart A.M., Klimek-Kopyra A., Boroń P.M. 2013. Morphological and molecular identification and PCR amplification to determine the toxigenic potential of Fusarium spp. isolated from maize ears in southern Poland. Phytoparasitica 41 (3): 241-248. DOI: https://doi.org/10.1007/s12600-012-0284-7

Lenc L., Lukanowski A., Sadowski C. 2008. The use of PCR amplification in determining the toxigenic potential of Fusarium sambucinum and F. solani isolated from potato tubers with symptoms of dry rot. Phytopathologia Polonica 48: 12-23.

Leslie J.F., Summerell B.A. 2006. The Fusarium Laboratory Manual. Blackwell Publish Ltd., UK, 388 pp.

Li H.P., Wu A.B., Zhao C.S., Scholten O., Löffler H., Liao Y.C. 2005. Development of a generic PCR detection of deoxynivalenol and nivalenol chemotypes of Fusarium graminearum. FEMS Microbiology Letters 243 (2): 505-511. DOI: https://doi.org/10.1016/j.femsle.2005.01.015

McMullen M., Jones R., Gallenberg D. 1997. Scab of wheat and barley: a reemerging disease of devastating impact. Plant Disease 81 (12): 1340-1348. DOI: https://doi.org/10.1094/ pdis.1997.81.12.1340

Miller J.D., Greenalgh R., Wang Y., Lu M. 1991. Trichothecene chemotype of three Fusarium species. Mycologia 83 (2): 121-130. DOI: https://doi.org/10.2307/3759927

Nash S.M., Snyder W.C. 1962. Quantitative estimations by plat counts of propagules of the Bean rot rot Fusarium in field soils. Phytopathology 52 (6): 567-572.

Nicholson P., Simpson D.R., Weston G., Rezanoor H.N., Lees A.K., Parry D.W., Joyce D. 1998. Detection and quantification of Fusarium culmorum and Fusarium graminearum in cereals using PCR assays. Physiological and Molecular Plant Pathology 53 (1): 17-37. DOI: https://doi.org/10.1006/ pmpp.1998.0170

Obanor F., Neate S., Simpfendorfer S., Sabburg R., Wilson P., Chakraborty S. 2013. Fusarium graminearum and Fusarium pseudograminearum caused the 2010 head blight epidemics in Australia. Plant Pathology 62 (1): 79-91. DOI: https:// doi.org/10.1111/j.1365-3059.2012.02615.x

Postic J., Cosic J., Vrandecic K., Jurkovic D., Saleh A.A., Leslie J.F. 2012. Diversity of Fusarium species isolated from weeds and plant debris in Croatia. Journal of Phytopathology 160 (2): 76-81. DOI: https://doi.org/10.1111/j.1439-0434. 2011.01863.x

Quarta A., Mita G., Haidukowski M., Santino A., Mulè G., Visconti A. 2006. Assessment of trichothecene chemotypes of Fusarium culmorum occurring in Europe. Food Additives and Contaminants 22 (4): 309-315. DOI: https://doi. org/10.1080/02652030500058361

Qu B., Li H., Zhang J., Xu Y., Huang T., Wu A., Zhao C., Carter J., Nicholson P., Liao Y. 2008. Geographic distribution and genetic diversity of Fusarium graminearum and F. asiaticum on wheat spikes throughout China. Plant Pathology 57 (1): 15-24. DOI: https://doi.org/10.1111/j.1365-3059. 2007.01711.x
Sampietro D.A., Ficoseco M.E.A., Jimenez C.M., Vattuone M.A., Catalán CA. 2012. Trichothecene genotypes and chemotypes in Fusarium graminearum complex strains isolated from maize fields of northwest Argentina. International Journal of Food Microbiology 153 (1-2): 229-233. DOI: https://doi.org/10.1016/j.ijfoodmicro.2011.10.029

Sampietro D.A., Marin P., Iglesias J., Presello D.A., Vattuone M.A., Catalan C.A.N. 2010. A molecular based strategy for rapid diagnosis of toxigenic Fusarium species associated to cereal grains from Argentina. Fungal Biology 114 (1): 74-81. DOI: https://doi.org/10.1016/j.mycres. 2009.10.008

Sarver B., Ward T., Gale L., Broz K., Kistler H.C., Aoki T., Nicholson P., Carter J., O’Donnell K. 2011. Novel Fusarium head blight pathogens from Nepal and Louisiana revealed by multilocus genealogical concordance. Fungal Genetic and Biology 48 (12): 1096-1107. DOI: https://doi.org/10.1016/j. fgb.2011.09.002

Somma S., Petruzzella A.L., Logrieco A.F., Meca G., Cacciola O.S., Moretti A. 2014. Phylogenetic analyses of Fusarium graminearum strains from cereals in Italy, and characterisation of their molecular and chemical chemotypes. Crop and Pasture Science 65 (1): 52-60. DOI: https://doi.org/10.1071/ cp13314

Waalwijk C., Kastelein P., Vries I. 2003. Major changes in Fusarium spp. in wheat in the Netherlands. European Journal of Plant Pathology 109: 743-754. DOI: https://doi. org/10.1007/978-94-017-1452-5_9

Ward T.J., Bielawski J.P., Kistler H.C., Sullivan E., O’Donnell K. 2002. Ancestral polymorphism and adaptive evolution in the trichothecene mycotoxin gene cluster of phytopathogenic Fusarium. Proceedings of the National Academy of Sciences, USA 99 (14): 9278-9283. DOI: https://doi. org/10.1073/pnas.142307199

Yang L., van der Lee T., Yang X.D., Waalwijk C. 2008. Fusarium populations on Chinese barley show a dramatic gradient in mycotoxin Profile. Phytopathology 98 (6): 719-722. DOI: https://doi.org/10.1094/phyto-98-6-0719

Yoruk E., Tunali B., Kansu B., Olmez F., Uz G., Zumrut I.M., Sarkaya A., Meyva G. 2016. Characterization of high-level deoxynivalenol producer Fusarium graminearum and F. culmorum isolates caused head blight and crown rot diseases in Turkey. Journal of Plant Diseases and Protection 123: 177-186. DOI: https://doi.org/10.1007/s41348-016-0027-y

Zhang H., van der Lee T., Waalwijk C., Chen W., Xu J., Jin Xu J.S., Zheng Y., Feng J. 2012. Population analysis of the Fusarium graminearum species complex from wheat in China show a shift to more aggressive isolates. PLoS One 7 (2): e31722. DOI: https://doi.org/10.1371/journal.pone.0031722

Zhang J.B., Li H.P., Dang F.J., Qu B., Xu Y.B., Zhao C.S., Liao Yu-C. 2007. Determination of the trichothcene mycotoxin chemotypes and associated geographical distribution and phylogenetic species of the Fusarium graminearum clade from China. Mycological Research 111 (8): 967-975. DOI: https://doi.org/10.1016/j.mycres.2007.06.008 